\title{
A Review of Research on Airline Passenger Volume Forecasting
}

\author{
Li Cheng $^{1 *}$, Xiao Mengting ${ }^{1}$ \\ 1 Shanghai University of Engineering Science, Shanghai 201620, China \\ licheng@sues.edu.cn (corresponding author), xdream_ting@163.com
}

\begin{abstract}
Based on the current research status both at home and abroad, this paper starts from two aspects of classic forecasting method (single forecasting method) and combined forecasting method, summarizes the existing research results of air passenger throughput forecasting. Therefore, it is instructive to find a prediction method that has more advantages for the prediction of air passenger traffic and how to improve the prediction accuracy better.
\end{abstract}

\section{Introduction}

The researches on the prediction of air passenger traffic are going on at home and abroad, and a series of research results have been obtained. However, the earlier studies mainly focused on the use of the regression method to establish the prediction model. Later, people found that using the combination forecasting method to predict the traffic volume with accuracy high, good fitting, and the combined forecasting method is obviously better than any one of the single forecasting method. Therefore, the research on air passenger traffic forecasting mainly starts from two major categories, one is from the typical forecasting method, that is, the single forecasting method, the other is from the perspective of portfolio forecasting, the weights are weighted by different ways of weighting. Based on the current research status both at home and abroad, starting with the above two aspects, this paper summarizes the existing research results of air passenger traffic forecast mainly according to a development course.

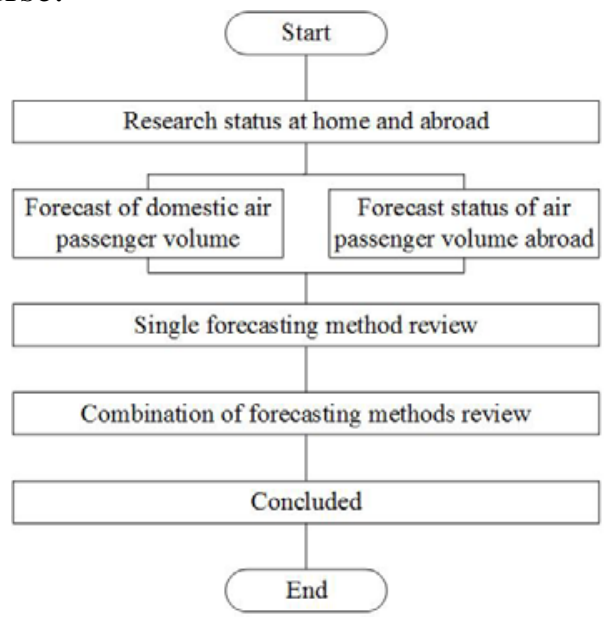

\section{AVIATION PASSENGER VOLUME FORECASTING LITERATURE REVIEW}

\subsection{Foreign Research Status}

Foreign air passenger traffic forecast earlier, but also made a series of research results. Early studies focused on the use of regression models to establish a predictive model. The factors to be considered include political, economic, cultural, customs and alternative modes of transportation. The regression analysis of these factors determines the corresponding elasticity of demand to reflect the relative trend of passenger traffic. Cigliano.J.M ${ }^{[1]}$ selected the GNP and fares as independent variables, the use of regression analysis of factors, respectively, the United States - Europe, Canada - Europe route passenger volume, studies have shown that: the two air routes passenger traffic on 
The gross national product is highly elastic, but the air traffic volume of Canada-Europe routes is less flexible than the price of the United States-Europe. Alperovich ${ }^{[2]}$ proves that the income demand of international air passenger transportation is relatively large and the price elasticity is relatively small, through the research on the forecast of international air passenger traffic volume. Bunn.D.W $\mathrm{W}^{[3]}$ predicts the transportation volume based on the combined forecasting method based on regression analysis, and concludes that the accuracy of the combined forecasting method is obviously higher than the single forecasting method. Dargay and Gately ${ }^{[4]}$ mainly studied the correlation between air passenger growth and revenue. After analyzing the historical data of large passenger traffic, they think there is a strong positive correlation between air passenger traffic and revenue.

Nowadays, many researches use neural network and fuzzy theory to predict it, and some achievements have been achieved. Using the neural network and regression analysis, Weigend ${ }^{[5]}$, Tung Bui and Jintae Lee ${ }^{[6]}$, Kim and Koehler ${ }^{[7]}$ respectively carried out the air passenger traffic forecasting. Based on the comparative analysis of the two forecast results, it is proved that the neural network Prediction method of prediction accuracy is better than the traditional statistical prediction. Based on the fuzzy time series analysis, Mohammad M Hamed, Hashem R Almasaeid, Zahi M Bani Said ${ }^{[8]}$, Lisoba $^{[9]}$ and Marie Cotterll ${ }^{[10]}$ established the fuzzy time series forecasting model of air passenger volume respectively. The regression prediction model and the time series model are comparatively analyzed. They think that the fuzzy time series model has higher prediction accuracy and stronger extrapolation prediction ability than the time series model.

\subsection{Domestic research status}

The forecast of domestic air passenger traffic started relatively late, but also achieved some results. Liu Jianhua and Ye Zhenwen ${ }^{[11]}$ choose GDP as the regression independent of the mode of transport, route mileage and number of passengers as regression independent variables, and establish the regression model of air passenger volume and the correlation analysis. The results show that GDP, airline mileage and passengers. The number is the main factor affecting the air passenger volume. Yang Song and Wang Wei ${ }^{[12]}$ used regression analysis to study the relationship between the growth of air traffic volume and GDP, pointing out that there is a strong correlation between the two. Ye Zhou, Li Zhongmin and Li Xiaofeng ${ }^{[13]}$ used the cointegration theory to study the cointegration relationship between aviation industry development and GDP growth. The shortterm and long-term relationship between them was analyzed by establishing the error correction model. They considered that the national economy. The promotion of the development of the aviation industry is significant, but the promotion of the aviation industry is not significant for promoting the growth of the national economy. Prof. Yang Hao ${ }^{[14]}$ from Beijing Jiaotong University analyzed the relationship between the economic development in the hinterland and the demand for transportation in "Integrated Transportation and Regional Economy". He believes that the economic development in the hinterland has a strong impetus to the transportation demand. Based on the research of regression prediction and artificial neural network, Cui Deguang, Wu Shining and $\mathrm{Xu}$ Bing ${ }^{[15]}$ of Tsinghua University made a proper combination and got the conclusion through empirical analysis that the forecasting accuracy of traffic volume High, good fitting, and the combined forecasting method is obviously better than any single forecasting method.

\section{A single forecasting method review}

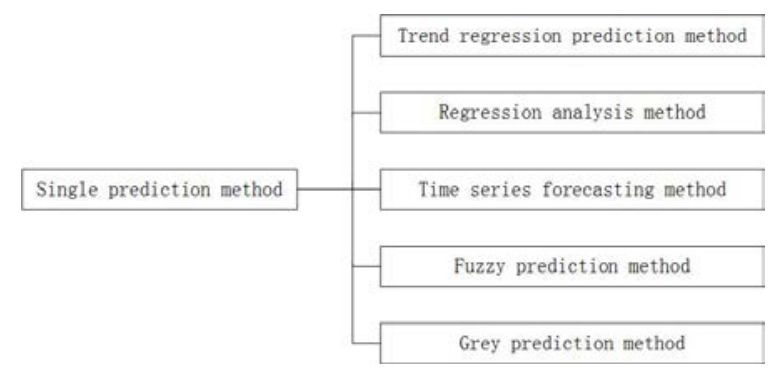




\subsection{Trend Regression Prediction Method}

Trend Regression Prediction ${ }^{[16]}$, also known as curve fitting, is by far the most studied and the most popular quantitative prediction method. Regression prediction method is mainly to study a kind of interdependence between a plurality of variables, through a large number of experiments and observations on each variable, and using an appropriate regression model to find the statistical regularity among the variables. However, in reality, the relationship between variables is complicated. Due to various factors, the relationship between these variables is very uncertain. Therefore, it is very difficult to establish the causal relationship between variables. The trend regression forecasting method does not need to look for the causal relationship between variables, but uses the time series as the independent variable of the regression model to fit a curve based on the historical data. The curve can reflect the trend of the forecasting quantity with time, then according to this trend to predict the future value. There are two or three basic assumptions that apply to trend regression prediction: the random error is irrelevant; the predictors are independent of the random error; and the predictors are linearly independent.

\subsection{Regression Analysis Method}

Regression analysis ${ }^{[17]}$ originated in the study of biology. In 1889 Golden's book Nature Genetics was published, the first of which was a regression analysis method, which was soon applied to the economy. The United States econometrician Moore first regression regression method to study the economy, has published many articles forecasting. Since then, regression analysis has been booming in economic forecasts.

\subsection{Time Series Forecasting Method}

Time series ${ }^{[18]}$ refers to a set of statistical data that explains the order of the number of variables in chronological order, whose values change over time and show some regularity in time. Time series prediction method is generally divided into deterministic time series and random time series. The application of deterministic time series has a long history. After World War II, the rapid development of computer technology has promoted the development of random time series. The time series forecasting method does not consider the causal relationship among the variables. Assuming that the change of the forecasting variables is only related with the time, it tries to simplify the forecasting by simplifying the complex and changeable factors related to the forecasting variables. Precisely because it does not take into account the causal relationship between the variables, it is not suitable for long-term prediction. At present, all countries in the world have taken it as one of the basic methods for forecasting short-term and medium-term economic growth. Commonly used time series forecasting methods are: trend regression forecasting method, trend moving average method, exponential smoothing method, autoregressive analysis and gray model.

\subsection{Fuzzy prediction method}

LAZadeh ${ }^{[19]}$ proposed the concept of fuzzy sets for the first time, and then proposed language variables and fuzzy algorithms. By adopting fuzzy algorithms to describe manual control rules described by former natural algorithms, LAZadeh eventually became a computer-usable algorithm. E.H.Mandani ${ }^{[20]}$ expands the fuzzy algorithm and proposes a fuzzy control method. Fuzzy control is a kind of nonlinear intelligent control method based on fuzzy set theory and fuzzy linguistic variables. Based on fuzzy inference, by imitating the human way of thinking, the control of the research object that is not easy to establish an accurate mathematical model is controlled. Sarat Kumar Patra and Bernard Mulgrew ${ }^{[21]}$ pointed out that the fuzzy prediction method has the advantage that the prediction results can be expressed in the form of prediction intervals and probabilities, and the prediction accuracy is high. The disadvantage is that the amount of data required is large and not suitable for the study of lean data objects. Since the 1970s, our country has also started the research in this area. Xie Jijian and Liu Chengping ${ }^{[22]}$ from Huazhong University of Science and Technology systematically elaborated fuzzy sets and their operations, fuzzy statistical methods, fuzzy clustering analysis, fuzzy model identification, fuzzy decision making, fuzzy linear 
programming, fuzzy control and their applications in science and technology and economy Management application and so on. Zhai Chunyan and Li Shichen ${ }^{[23]}$ systematically expounded the fuzzy predictive control algorithm, and analyzed its optimization method in detail, and pointed out the future development direction of fuzzy predictive method.

\subsection{Gray prediction method}

Professor Deng Julong ${ }^{[24]}$ first proposed the product of the concept of a gray-system forecast that can be used both for socioeconomic forecasting and for industrial control as a cybernetic point of view and methodological extension. System refers to two or more interacting elements posed by a specific function of an organic whole, according to the visibility of information, the system can generally be divided into white, gray and black three. The gray system rearranges the original data sequence by selecting the appropriate generation method and seeks its internal regularity. The key of the gray prediction method is how to choose the appropriate method to find this regularity, that is, through which generation algorithm to generate a new data sequence in order to better weaken its randomness, highlighting its regularity.

\section{A combination of forecasting methods review}

Combination forecasting is not a new forecasting method, but a method to improve the accuracy of the existing forecasting results. In view of this method is simple and effective, has been widely used. Combined forecasting is called Combination Forecasting or Combined Forecasting in foreign countries. In recent decades, combined forecasting has become a hot topic in forecasters' field of study. It will be the focus of attention in the future research. In recent years, Chinese scholars have also made a series of important achievements in the research of portfolio forecasting. Yang Guangxi ${ }^{[25]}$ using the combination forecasting method of economic forecast, proved that the accuracy of the combined forecasting method is obviously better than the single forecasting method. Based on the absolute error of the combined model, Tang Xiaowo and Ma Yongkai ${ }^{[26]}$ studied the optimization of linear combination forecasting model and provided a reference for improving the prediction accuracy. Ma Yongkai, Tang Xiaowo and Yang Guiyuan ${ }^{[27]}$ studied non-negative weight optimal combination forecasting methods from many aspects and proposed a simple method to solve the third order non-negative weight optimal portfolio forecasting problems.

In 1954, the United States entered Schmitt to predict the population of 37 major cities in the United States by using the combined forecasting method, which improved the forecasting accuracy. The first systematic and comparative study of combinations was the paper "Combination of Predictive Values" by J.M.Bates and C.W.J .Granger (1969), but it was limited to a combination of two predictors ${ }^{[28]}$. In 1989, Journal of Forecasting, an authoritative academic journal in the field of international forecasting, published a portfolio forecasting album, which fully illustrated the importance of portfolio forecasting in forecasting research. In recent years, our country also made a series of research results in the research of portfolio forecasting methods. Professor Tang Xiaowo from the University of Electronic Science and Technology of China (now Deputy Director of Education Department of Sichuan Province) and Professor Cao Changxiu of Chongqing University have made outstanding achievements. One after another in the field of forecasting, the authoritative academic journals "Prediction", "Journal of Management Engineering", "Finance and Economics", "Investment Theory and Practice", "Journal of Electronic Science and Technology" and other journals published a series of academic papers on portfolio forecasting, to promote our theoretical research and application of portfolio forecast has made important contributions.

At present, domestic and foreign scholars mainly propose the following methods in portfolio forecasting: minimum variance, Kalman filtering, unconstrained least squares, Bayesian, variable weight combination forecasting, recursive equal portfolio forecasting, performance advantages Matrix method and so on. Among the above-mentioned various combined forecasting methods, the least-variance method and its expansion are mostly studied theoretically and practically. At present, the properties of the minimum variance method, the features of the composite structure and the range of the square of the prediction error have not been studied yet. The solution of these problems is of 
great significance for the study of combinatorial prediction methods.

Spyros and Robert ${ }^{[29]}$ have done a large amount of empirical analysis on the simple average method, that is, the effectiveness of the EW method. Kong Qingkai ${ }^{[30]}$ pointed out the application conditions of the EW method. Wang Yu and Liu Bao ${ }^{[31]}$ proposed on the basis of the EW method The Recursive Equality Combination Prediction Method is REW method, and an example is given to prove the validity of REW method.

Zhou Zongfang, Yang Chunde ${ }^{[32]}$ proposed a new method for determining the weight of combined forecasting, that is, using the target programming method to determine the combined forecasting weights. They believe that due to the increasing complexity of the environment and the continuous improvement of their decision-making ability, a large number of facts show that the actual development results of things are always more or less influenced by social environmental factors and personal awareness of policymakers, and even some emergencies There is no absolute accurate and reliable forecasting method. The method proposed in this article emphasizes the awareness of decision-makers' participation and makes use of the flexibility of the target programming method to expand the scope of the portfolio forecasting weights.

Cao Changxiu ${ }^{[33]}$ and Tang Xiaowo et al. ${ }^{[34]}$ proposed two fuzzy variable weight combination forecasting methods. However, the weighting method proposed by Cao Changxiu at present weights only depends on the error of the previous moment and the rate of change of error, so this method has some limitations. Tang et al. Have improved on this. The weight of the current moment is determined according to the relative index of the absolute average of the prediction error in the recent period and the relative index of the absolute cumulative error of the prediction error in the limited rolling-time domain length. Variable weight combination forecasting method needs further study. To improve the accuracy of combined forecasting, we must study the variable weight combined forecasting method.

Chen Huayou, Liu Chunlin, Sheng Zhaohan ${ }^{[35]}$ An Ordered Weighted Weighted Average (OWHA) operator is proposed. Based on this, a combined forecasting model is proposed to induce Ordered Weighted Weighted Average (IOWHA) operators. Wu Liang-ping, Zhang Jian, Lu Yuan ${ }^{\text {[36] }}$ based on the previous work, the combination forecasting model based on IOWHA operator was applied to inbound tourism in China and achieved good results. Chen Huayou, Sheng Zhaohan ${ }^{[37]}$ a non-linear combination forecasting model of induced ordered weighted geometry (IOWGA) operator was established. Chen Huayou, Sheng Zhaohan, Liu Chunlin ${ }^{[38]}$ On the basis of previous studies, we define the generalized weighted arithmetic average combination forecasting superiority and redundancy based on the concept of P-power error, discuss the optimal theoretical basis and its Mathematical properties. Chen Huayou, Li Xiang, Jin Lei and Yao Mengjie ${ }^{[39]}$ put forward a combination forecasting model based on density operator. The model is characterized by the density of distribution and the accuracy of forecasting

TABLE I.

\begin{tabular}{|l|l|}
\hline \multicolumn{1}{|c|}{ Researcher } & Combination of forecasting methods \\
\hline Spyros and Robert & EW methods \\
\hline Wang Yu, Liu Bao & REW methods \\
\hline Zhou Zongfang, Yang Chunde & Goal programming method \\
\hline Cao Changxiu et al. and Tang Xiaoli et al. & Fuzzy variable weighting method \\
\hline $\begin{array}{l}\text { Chen Huayou, } \\
\text { Liu Chunlin, } \\
\text { Sheng Zhaohan }\end{array}$ & IOWHA operator \\
\hline $\begin{array}{l}\text { Chen Huayou, } \\
\text { Sheng Zhaohan }\end{array}$ & IOWGA operator \\
\hline $\begin{array}{l}\text { Chen Huayou, } \\
\text { Sheng Zhaohan, } \\
\text { Liu Chunlin }\end{array}$ & $\begin{array}{l}\text { Prediction method based on vectorial angle } \\
\text { cosine }\end{array}$ \\
\hline $\begin{array}{l}\text { hen Huayou, Li Xiang, } \\
\text { Jin Lei and Yao Mengjie }\end{array}$ & Prediction method based on density operator \\
\hline
\end{tabular}




\section{Conclusion}

The combination prediction method has the advantages of high accuracy and good fitting for the air passenger traffic forecasting, and the prediction result of the combined forecasting method is obviously superior to any one of the single forecasting methods. However, the combination forecasting is essentially a weighted average of the individual forecasting methods according to the error size, so that the combined forecasting model results have more accurate and reliable effects than the single forecasting methods. Research has also made a lot of research results.

However, in the research of air passenger traffic, the complexity of the research object sometimes cannot be accurately expressed by a single value, and many influencing factors show a dynamic range interval, so interval time series prediction and measurement criterion can be considered. Interval type combination prediction is essentially Is based on the nature of the interval number, combined with the combination of forecasting methods to predict, in order to have a more accurate estimate of the interval number, so as to better deal with the uncertainty of the phenomenon, the domestic research on interval-type prediction also made a lot of research results, If used in traffic volume prediction, it can overcome the disadvantage that the traditional combined forecasting method gives invariable weighted average coefficient and the single error index as the prediction accuracy.

\section{Acknowledgment}

Foundation Project: National Social Science Foundation Project (No.15BJL104).

Correspondence Author: Li Cheng (1980- ), doctor, associate professor, research field is management engineering, civil aviation management.

\section{References}

[1] Cigliano J.M. A Meta-Analytic Review of International Tourism Demand [J]. Journal of Travel Research, 1999, 37(3):273-284

[2] Alperovich G A. The size distribution of cities: on the empirical validity of the rank-size rule [J]. Journal of Urban Economics, 1984, 16: 232-239

[3] Bunn D. W. Combining forecasts [J]. European Journal of Operational Research, 1998, 33 (3): 223-229

[4] Dargay J, Gately D. Income's effect on vehicle ownership worldwide: 1960-2015[J]. Transportation Research (Part A), 1999, 33:101-138

[5] Weigend A Betal. Predicting the future: a connectionist approach [J]. Intl.J.Neur. Sys, 1990(1): 198-209

[6] Tung Bui, Jintae Lee. An agent-based framework for building decision support systems [M].Decision Support System, 1999, 25(3): 226-234

[7] Kim H, Koehler G J. Theory and practice of decision tree induction [J]. Omega, 1995, 23 (6): 644-652

[8] Mohammad M Hamed, Hashem R A1 masaeid, Zahi M Bani Said. Short term Prediction of Traffic Volume in Urban Arterials [M].Journal of Transportation Engineering, 1995

[9] Lisoba PGJ. Neural network-current applications [M]. London: Chapman\&Hall, 1992.

[10] Marie Cotterll, et al. Foereasting of Curves Using a Kohonen Classification [J]. Journal of Forecasting, 1998(17): 434-439

[11] Liu Jianhua, Ye Zhenwen. China's civil aviation passenger transport empirical research [J]. China Civil Aviation Flight Journal, 2002 (4): 34-37

[12] Yang Song, Wang Wei. Research on the Relationship between the Growth of Air Transport 
Volume and GDP Growth [J]. China Civil Aviation, 2006 (1): 46-49

[13] Ye Zhou, Li Zhongmin, Li Xiaofeng. An Empirical Analysis on the Relationship between the Development of Civil Aviation in China and the Growth of National Economy [J]. Journal of Tianjin Polytechnic University .2005,21 (5): 81-84

[14] Yang Hao. Integrated transport and regional economy [M]. Beijing: China Railway Publishing House, 1995 (1)

[15] Cui Deguang, Wu Shining, Xu Bing. Journal of Tsinghua University, 2005,46 (1): 96-99 Comment for this article: Feedback Author Email Title Code Content Copyright by Journal of Tsinghua University

[16] Tao Jingxuan. Economic Prediction and Decision [M]. Beijing: China Metrology Press, $2004(1)$

[17] Feng Wenquan. Economic forecasting and decision-making technology [M]. Wuhan: Wuhan University Press, 2002 (3)

[18] Zhang Guixi. Economic Prediction, Decision-Making and Countermeasures [M] .Beijing: Capital University of Economics and Business Press, 2003

[19] LA Zadeh. Fuzzy sets [J]. Information and Control, 1965, 8 (3): 338-353

[20] E.H.Mamdani. Applications of fuzzy algorithms for simple dynamic plants [J].Proc.IEE, 1974(121): 1585-1588

[21] Sarat Kumar Patra and Bernard Mulgrew. Fuzzy techniques for adaptive nonlinear equalization .Signal Processing, 2000, 80 (6): 985-998

[22] Xie Jijian, Liu Chengping.Fuzzy mathematical method and its application [M]. Wuhan: Huazhong University of Science and Technology Press 2006 (3)

[23] Zhai Chunyan, Li Shichen.Fuzzy Prediction Control Algorithm [J]. Process Automation Instrumentation, 2004,025 (3): 1-4

[24] Deng Julong. Gray System Theory [M]. Wuhan: Huazhong University of Science and Technology Press, 1990

[25] Yang Guangxi.Application of portfolio forecasting method in economic forecast [J]. Statistics and Prediction, 1998 (6): 17-20

[26] Ma Yongkai, Tang Xiaolu.Study on optimization of linear combination forecasting model [J] .Systems Engineering -Theory \& Practice, 1998,18 (2): 111-114

[27] Ma Yongkai, Tang Xiaoli, Yang Guiyuan.Study on the Basic Theory of Non-negative Weight Optimal Combination Forecasting Method [J]. Operations Management and Management, 1997, 26 (3): 290-291

[28] J.M.Bates and C.W.J.Granger, Combination of Forecasts [J].Journal of Forecasting.1969(6)

[29] Spyros Markridarkis and Robert L. Winkler, Averages of Forecasts: Some Emprical Results. Management Sciences, V01.29, No. 9,1983

[30] Kong Qing Kai. Application of the average forecasting method [J]. Forecast, 1985 (5)

[31] Wang Yu, Liu leopard. Recursive equal weight combination forecast [J]. Forecast, 1988 (5)

[32] Zhou Zongfang, Yang Chunde. Application of target programming to determine the combination of the number of predictive weight [J]. Journal of Chongqing University of Posts and Telecommunications, 1994 (2): 59-64

[33] Cao Changxiu, Wang Jing, Tang Xiaolu. A Fuzzy Variable Weight Combinatorial Prediction Method - FVW Method [J]. Prediction, 1996 (5): 49-50 
[34] Don himself, Wang Jing, Cao Changxiu. A New Fuzzy Adaptive Variable Weight Combination Forecasting Method [J]. University of Electronic Science and Technology of China, 1997,26 (3): 289-292

[35] Chen Huayou, Liu Chunlin, Sheng Zhaohan. IOWHA Operator and Its Application in Combination Forecasting [J]. Chinese Journal of Management Science, 2004,12 (5): 36-41

[36] Wu Liangping, Zhang Jian, Lu Yuan. Application analysis of portfolio forecast based on IOWHA operator in China inbound tourism [J]. Journal of Tourism, 2011,26 (11): 19-27

[37] Chen Huayou, Sheng Zhao Han. A New Kind of Combined Forecasting Method Based on IOWGA Operator [J]. Journal of Management Engineering, 2005,19 (4): 36-39

[38] Chen Huasheng, Sheng Zhaohan, Liu Chunlin. Study on the Properties of Combined Forecasting Model Based on Vector Cosine Angle Cosine [J]. Journal of Management Science, 2006,9 (2): 1-8

[39] Chen Huayou, Li Xiang, Jin Lei, Yao Mengjie. Interval combination forecast method based on correlation coefficient and IOWA operator [J]. Statistics and Decision, 2012,27 (6): 83-86 